ÉGYPTE monde arabe

\section{Égypte/Monde arabe}

$21 \mid 1995$

Économie égyptienne et perspectives de paix au Proche-Orient

\title{
Le système bancaire égyptien et la politique d'ajustement structurel
}

\section{Salwa al-Antary}

\section{(2) OpenEdition}

\section{Journals}

Édition électronique

URL : https://journals.openedition.org/ema/397

DOI : $10.4000 /$ ema.397

ISSN : 2090-7273

Éditeur

CEDEJ - Centre d'études et de documentation économiques juridiques et sociales

Édition imprimée

Date de publication : 31 mars 1995

Pagination : 156-161

ISSN : 1110-5097

Référence électronique

Salwa al-Antary, « Le système bancaire égyptien et la politique d'ajustement structurel », Égypte/ Monde arabe [En ligne], 21 | 1995, mis en ligne le 08 juillet 2008, consulté le 07 juillet 2022. URL : http:// journals.openedition.org/ema/397 ; DOI : https://doi.org/10.4000/ema.397

Ce document a été généré automatiquement le 7 juillet 2022

Tous droits réservés 


\title{
Le système bancaire égyptien et la politique d'ajustement structurel
}

\author{
Salwa al-Antary
}

1 Cette étude se propose d'analyser le rôle du système bancaire dans la politique de réforme économique et d'ajustement structurel adoptée par l'Égypte, conformément aux accords conclus en 1991 avec le FMI et la Banque mondiale. Nous analyserons en particulier dans quelle mesure le secteur bancaire a contribué à permettre à l'économie égyptienne de s'acquitter du service de la dette et à restaurer sa solvabilité ${ }^{1}$, objectif final de ces accords.

Les principales caractéristiques de la politique monétaire

2 La politique déflationniste repose sur la baisse du taux de change, la hausse des taux d'intérêt et le contrôle des liquidités par émission de bons du Trésor à taux variables. Les banques égyptiennes doivent respecter les normes internationales du capital adequacy $^{2}$. Quant aux banques étrangères, elles ont obtenu le droit d'opérer en livres égyptiennes, et les sociétés du secteur public celui de traiter avec toutes les banques, publiques ou privées, locales ou étrangères.

Politique des devises

3 Conformément à l'accord conclu avec le FMI, la Banque centrale d'Égypte s'est engagée à consolider ses réserves en devises selon un calendrier trimestriel ${ }^{3}$. Cette consolidation a été favorisée par une conjoncture exceptionnelle. En effet, la guerre du Golfe, puis le séisme d'octobre 1992, et enfin l'annulation d'une partie de la dette extérieure égyptienne, se sont traduits par un flux important de transferts officiels. Mais c'est l'augmentation du taux d'intérêt sur les dépôts en livres égyptiennes, relativement à ceux des autres devises, qui a permis d'attirer à l'étranger une partie de l'épargne égyptienne, transformée en comptes d'épargne bancaires ou en bons du Trésor. La Banque centrale d'Égypte s'est ainsi instituée en acheteur sur le marché des changes afin de consolider ses réserves en devises et de maintenir le cours de la livre égyptienne à un niveau suffisamment bas pour garantir les rentrées de devises du système bancaire officiel. Les réserves nettes en devises de la Banque centrale ont augmenté de 6,3 milliards de dollars à la fin mars 1991 à 17 milliards de dollars à la fin 
juin $1994^{4}$. C'est ainsi que, pour la première fois depuis le début du rééchelonnement de la dette extérieure égyptienne en 1987, la Banque centrale a pu mobiliser suffisamment de devises pour couvrir le service de la dette rééchelonnée (cf. tableau 1). 86\% de ces réserves ont été placées à court terme à l'étranger. Les $14 \%$ restants ont servi à l'acquisition de titres étrangers, constitués pour la plupart de bons du Trésor américain. Le montant de ces titres est passé de 0,5 à 7,5 milliards de livres égyptiennes entre juin 1990 et juin 1993 pour retomber à 4,9 milliards en juin 1994. Leur gestion est confiée, sous contrat. à la Federal Reserve Bank of New York ${ }^{5}$. La part des dépôts en devises du système bancaire placés sur les marchés internationaux est ainsi passée de 60\% en juin 1990 à 85\% en juin 1994. La baisse de la part des placements en Égypte dans l'épargne locale en devises témoigne, pour partie, de la stagnation due aux politiques déflationnistes. Ainsi, les avoirs des banques égyptiennes sur l'extérieur n'ont cessé de croître, passant de 11,2 à 73,4 milliards de livres égyptiennes (LE) de juin 1990 à juin 1993, puis atteignant 84,17 milliards LE en juin 1994. Le système bancaire égyptien, Banque centrale comprise, a donc placé sur le marché international, à la fin de la première phase de la politique de réforme, l'équivalent de 24,8 milliards de dollars, correspondant à 83\% de la dette extérieure de l'Égypte au 30 Juin 1994.

Le mode de placement des réserves

4 Si une part notable de l'épargne est placée à l'étranger (19\% des actifs des banques égyptiennes en juin 1993), le mode d'investissement en Égypte a été profondément modifié sous l'effet des politiques monétaires et fiscales déflationnistes. L'achat, par les banques, de bons du Trésor est passé de 3,1 à 28,9 milliards de livres entre juin 1991 et juin 1994, absorbant $18 \%$ du total de leurs placements locaux à cette date (cf. tableau 3). Ce placement au rendement élevé et sans risque résolvait le double problème de la faiblesse de la demande de crédit et des restrictions imposées à l'octroi des prêts. Ces restrictions comprennent l'obligation de relever les provisions pour créances douteuses et l'application aux dettes du secteur public de critères de risque identiques à ceux pratiqués pour les créances sur le secteur privé. En conséquence, la part des créances dans l'actif bancaire a diminué de 61 à 50\%. le rapport crédits/dépôts baissant également de $71 \%$ à $57 \%$ entre juin 1990 et juin 1994. Les prêts à la consommation (de véhicules de transport et de biens de consommation durables) sont apparus comme une autre solution a l'excédent d'épargne, faisant passer la part des ménages dans le total des crédits locaux de $3 \%$ à $12 \%$ sur la même période ${ }^{6}$. Le volume total des financements (sous forme de crédits ou de participation directe au capital) concédés par les banques au secteur privé en juin 1994 s'élevait à près de 42,9 milliards de livres, contre 24 milliards pour les entreprises publiques ${ }^{7}$.

5 La tendance longue au déplacement du crédit bancaire du public vers le privé a.été renforcée par la réforme. En effet, la part des prêts accordés au secteur public a chuté d'un plafond de $68 \%$ en juin 1980 à un plancher variant entre $24 \%$ et $28 \%$ entre juin 1990 et juin 1993. La loi 203 de 1991 sur les entreprises publiques a supprimé l'obligation, pour l'État, de garantir leurs dettes et de renflouer leurs déficits. Elle interdit également aux holdings de leur octroyer des prêts. Au même moment, et conformément à l'accord conclu avec la Banque mondiale ${ }^{8}$, le risque lié aux opérations avec le secteur étatique était relevé. Par ailleurs, la distinction entre public et privé, accordant au premier un plafond de crédits supérieur au second, était annulée, renforçant la compétition entre les deux secteurs. Le recours croissant au découvert bancaire, conséquence de la brutale réduction dès crédits octroyés, a accentué l'endettement des entreprises publiques. C'est ainsi que la loi 203, la nouvelle politique 
monétaire et la gestion du système bancaire ont contribué à aggraver la crise financière du secteur étatique, ne laissant d'autre issue que la privatisation. Cette privatisation se manifeste sous plusieurs formes, allant de la simple location de lignes de production à la vente partielle ou intégrale du capital des entreprises publiques.

Le rôle des banques dans la privatisation

Depuis 1990, le gouvernement égyptien a commencé à mettre en vente les avoirs du secteur étatique. En pleine négociation avec le FMI et la Banque mondiale pour l'annulation de $15 \%$ de la dette extérieure de l'Égypte, il annonçait, en janvier 1993, son programme détaillé de privatisation. Ce dernier permettait aux Egyptiens et aux étrangers d'acheter sans limite maximum ces avoirs, excepté dans le Sinaï, où seuls les Egyptiens sont autorisés à acquérir des actifs dans les entreprises et les hôtels. Privatisation ouverte au capital étranger et conversion de la dette en prises de participation allaient de pair. A la suite de cette initiative, le capital étranger a exercé de nombreuses pressions pour acquérir la première tranche des avoirs offerts à la vente. Le gouvernement égyptien avait restreint l'acquisition des trois premières sociétés privatisables - Coca-Cola, Pepsi-Cola et l'Hôtel Shephard - à un seul acheteur. Grâce à leurs moyens financiers considérables, mais aussi en raison du chantage au retrait des concessions et à l'interruption de la fourniture des intrants nécessaires à la production, les deux firmes transnationales américaines ont réussi à écarter les repreneurs égyptiens potentiels. La Société Heinan International, gérante de l'Hôtel Shephard, a exploité à son profit une clause du contrat de gestion pour empêcher la vente de l'hôtel à un tiers. Selon cette clause, le gouvernement égyptien s'interdisait de prendre toute mesure susceptible de réduire l'autonomie de gestion de la société gérante. Le système bancaire égyptien contribue au processus de privatisation comme intermédiaire dans les opérations de vente et de conversion de la dette extérieure. Il gère la vente partielle ou totale des sociétés publiques, participant, avec les capitaux étrangers, à l'évaluation des entreprises privatisables. Cette participation se traduit par la prise en charge des opérations de vente, la garantie de la souscription aux actions ou le financement des achats, et ce jusqu'à $70 \%$ de la valeur des titres vendus ${ }^{9}$. On peut se demander si ce financement va s'étendre aux acheteurs étrangers, l'épargne locale finançant par là même l'achat des entreprises publiques par le capital international. Par ailleurs, les banques commerciales étatiques cèdent progressivement leurs parts dans les sociétés d'État ou en augmentent le capital par souscription publique ${ }^{10}$. Elles sont censées vendre, selon l'accord passé avec le FMI et la Banque mondiale, actuellement et jusqu'en juin 1995, leurs parts dans les capitaux des banques mixtes en attendant leur propre privatisation ${ }^{11}$.

Perspectives de l'activité bancaire dans le cadre de la réforme

7 L'activité future du système bancaire égyptien se laisse deviner aux nouvelles opérations que les banques mènent, soit directement, soit en créant des sociétés spécialisées : constitution de portefeuilles de titres étrangers sur les marchés internationaux, courtage, gestion de patrimoine de la clientèle et autres services boursiers; fonds communs de placement; opérations de change à terme; commerce immobilier; commercialisation locale et exportation du coton. La spécialisation du capital bancaire égyptien vers les placements financiers et la spéculation aux dépens des activités productives pourrait ainsi s'accentuer avec l'ajustement structurel.

Conclusion 
D'une manière générale, le système bancaire égyptien a réussi Jusqu'ici à réaliser l'objectif de la politique monétaire du programme d'ajustement structurel, à savoir rendre l'économie égyptienne solvable vis-à-vis de ses créancière internationaux. $\mathrm{H}$ a su en effet mobiliser l'épargne en devises et consolider les résolves de la Banque centrale, assurant par-là même le service de la dette. Il a permis au capital international d'acquérir une partie des capacités productives égyptiennes privatisées par le canal de la conversion de la dette en

prise de participation. En contrepartie, le rétablissement de la solvabilité de l'Égypte s'est effectué à un coût élevé pour l'économie nationale. En effet, une part croissante de l'épargne égyptienne en devises est placée a l'extérieur, tandis que l'épargne locale est détournée de l'investissement vers les bons du Trésor et la spéculation. Quant au secteur public, qui fournit $40 \%$ du produit intérieur brut, sa crise financière a été fortement accentuée.

10 A la lumière de ces résultats, la question reste de savoir dans quelle mesure ces évolutions peuvent favoriser un développement économique et social permettant à l'économie égyptienne d'accéder à une croissance autonome.

11 Traduit de l'arabe par Mona Niazi

\section{ANNEXES}

Tableau 1 : Evolution de l'actif de la Banque centrale en devises et engagements découlant du service de la dette (en milliards LE)

\begin{tabular}{|l|l|l|}
\hline Date (fin juin) & Actifs nets & Engagements \\
\hline \hline 1987 & 1.508 & 1.385 \\
\hline \hline 1988 & 1.303 & 5.016 \\
\hline \hline 1989 & 1.360 & 8.852 \\
\hline \hline 1990 & 2.329 & 15.771 \\
\hline \hline 1991 & 17.677 & 33.646 \\
\hline \hline 1992 & 30.699 & 38.905 \\
\hline \hline 1993 & 50.231 & 38.792 \\
\hline \hline 1994 & 58.142 & 40.063 \\
\hline
\end{tabular}


Source : Banque centrale d'Égypte, Rapport annuel 1992/93, pp. 167-169, et Rapport présenté au parlement, 1993/94, pp. 73 à 77.

Tableau 2 : Balance nette des opérations du système bancaire égyptien avec les banques établies à l'étranger (en milliards LE)

\begin{tabular}{|l|l|l|l|l|l|}
\hline & 1990 & 1991 & 1992 & 1993 & 1994 \\
\hline \hline Banque centrale & 2,80 & 17,22 & 25,25 & 42,77 & 53,53 \\
\hline \hline Banques commerciales & 10,00 & 16.01 & 23,07 & 22,63 & 24,49 \\
\hline \hline Banques d'investissement & 3,97 & 6,85 & 4,95 & 7,85 & 6,01 \\
\hline \hline Banques spécialisées & 0,06 & 0,05 & $.0,02$ & $.0,11$ & 0,14 \\
\hline \hline Total & 11,23 & 40,13 & 53.47 & 73.36 & 84,17 \\
\hline
\end{tabular}

Source : Chiffres établis sur la base des données de la Banque centrale : Rapport annuel 1992/93, Annexe statistique. Evolution des crédits et des activités bancaires de juillet 1990 à juin 1992 et 1993/94.

Tableau 3 : Répartition des placements du système bancaire égyptien à l'intérieur du pays (en milliards LE)

\begin{tabular}{|c|c|c|c|c|c|c|c|c|c|c|}
\hline & \multicolumn{2}{|l|}{1990} & \multicolumn{2}{|l|}{1991} & \multicolumn{2}{|l|}{1992} & \multicolumn{2}{|l|}{1993} & \multicolumn{2}{|l|}{1994} \\
\hline & LE & $\%$ & LE & $\%$ & LE & $\%$ & LE & $\%$ & LE & $\%$ \\
\hline Titres et participations & 8.5 & 11 & 16,9 & 16 & 34,9 & 30 & 46,6 & 33 & 49.2 & 31 \\
\hline - Bons du Trésor & - - & - & 3.1 & 3 & 2,6 & 11 & 23,5 & 17 & 28.9 & 18 \\
\hline -Autres titres d'Etal & 6,3 & 8 & 1,3 & 11 & 9,6 & 17 & 19,4 & 14 & 15.8 & 10 \\
\hline Comptes en banque & 21.4 & 28 & 30,6 & 29 & 29,0 & 24 & 29.3 & 21 & 30,6 & 19 \\
\hline Crédits & 46,5 & 61 & 57,7 & 55 & 54,6 & 46 & 65,1 & 46 & 78,1 & 50 \\
\hline Total & 76,4 & 100 & 105.2 & 100 & 118,5 & 100 & 141,0 & 100 & 157,9 & 100 \\
\hline
\end{tabular}

Source : Tableau établi sur la base des données de la Banque centrale d'Égypte, Rapport annuel 1992/93, Annexe statistique. Evolution des crédits et des activités bancaires de juin 1990 à juin 1992; tableaux relatifs à la répartition de la valeur comptable des titres et des investissements des banques.

NB : Faute de données détaillées publiées pour l'année 1993/94, nous avons supposé constant le montant des placements financiers à l'extérieur durant l'année 1992/93. 


\section{NOTES}

1. Banque mondiale, Egypt, Financial Policy for Adjustment and Growth, vol. 3, septembre 1993, pp. 54-60.

2. Selon une décision du comité de Baie, le ratio fonds propres/actifs totaux des banques ne devait pas être inférieur à un taux minimal de $8 \%$ affecté d'un coefficient de risque relatif à la structure des actifs.

3. Korayem K., Structural Adjustment and Reform Policies, United Nations Economic and Social Council, 1993, pp. 15-16.

4. Banque centrale d'Égypte, Rapport annuel 1992/93, p. 88.

5. Banque mondiale, op. cit., p. 81.

6. Banque centrale d'Égypte, op. cit.

7. Chiffres établis à partir des données de la Banque centrale d'Égypte (Rapport annuel de 1980-81,1984/85, 1992/93. Evolution du crédit et de l'activité bancaire juillet 1980-jum 1984 et Juillet 1990-Juin 1992).

8. Chakir F. (directeur général du contrôle sur les banques, Banque centrale d'Égypte), «Les causes de la réforme bancaire en Égypte », séminaire sur $L a$ réforme bancaire dans le monde arabe, Union des banques arabes. Amman, Jordanie, avril 1993.

9. Banque nationale d'Égypte, Bulletin économique $n^{\circ} 1 \& 2,1993$, p. 25.

10. En 1993, le capital de la Commercial International Bank a été augmenté au moyen de la souscription publique et la Banque nationale d'Égypte n'a pas participé à celle augmentation, ce qui a fait baisser sa part de $70 \%$ à $43 \%$. Cinq pour cent du capital de la Banque est échu à la Corporation financière internationale qui relève de la Banque mondiale.

11. Al-Rifai F. (vice-gouverneur de la Banque centrale), the Banker, a Financial Tunes Publication, Londres, juin 1993, p. 51.

\section{INDEX}

Mots-clés : ajustement structurel, système bancaire

\section{AUTEUR}

\section{SALWA AL-ANTARY}

Directrice adjointe des recherches, Banque nationale d'Égypte 